\title{
Study on islanding detection methods for distributed system
}

\author{
Bo-Han Liu†, Li Bo, Guo-Zeng Yan, Xiang-Ping Ni and Zhen-Tao Liu \\ State Grid AC Engineering Construction Company, Xicheng District, Beijing 102206, \\ China \\ ${ }^{\dagger}$ E-mail:ncmomo@163.com
}

\begin{abstract}
Islanding in power system can be intentional or unintentional. In the past most of the methods were developed for the phenomenon of unintentional islanding. Recently as the distributed generator (DG) sources are able to control voltage and frequency in the islanded part, the phenomenon of unintentional islanded is not common. If the autonomous operation of sources or intentional islanding is desired, then a fast islanding detection technique is necessary. The main philosophy of detecting an islanding situation is to monitor the DG output parameters and system parameters to decide whether or not an islanding situation has occurred from change in the parameters. islanding detection techniques can be classified as remote techniques and local techniques, and local techniques can be divided into passive methods, active methods and hybrid methods. In this paper, several remote and local techniques (passive, active, and hybrid methods) for islanding detection are briefly described. The advantage and limitation of each technique are also introduced. Meanwhile, the comparison of these methods is also discussed.
\end{abstract}

Keywords: remote detection method; local detection methods; hybird method; islanding; DG.

\section{Remote Islanding Detection Methods}

Remote islanding detection methods are based on communication between utilities and DGs. Although these techniques may have better reliability than local techniques, they are expensive to implement and hence uneconomical. Some of the remote islanding detection techniques are as follows:

\subsection{Transfer trip scheme}

The transfer trip scheme is the most direct method for loss of grid protection and is achieved by monitoring of auxiliary contacts on all circuit breakers on the utility system between its main sources of generation and the dispersed storage and generation units $[1,3]$. The basic idea of this method is to monitor the status of all the circuit breakers and reclosers that could island a distribution system. When a switching operation produces a loss of grid, a transfer trip scheme can then be used to open the inter-tie connecting the two systems. Following successful restoration of the utility supply, the dispersed storage and generation 
units can be resynchronised to the utility and then reconnected. Unfortunately, several circuit breakers are candidates for creating the loss of grid and a comprehensive monitoring system involving all circuit breakers which could be involved would be unmanageable for most utilities. The installation of an extensive Supervisory Control and Data Acquisition (SCADA) system and network automation can facilitate such a scheme [4]. This method requires a better interaction between the utility and DGs and this often increases the costs for both the utility and DG owners. In addition to high cost, the transfer trip scheme could be very complicated as well, for example, each openable device between DG and supply system needs a transmitter. Some of the devices need to be reconfigured and equipped with the capability of interfacing with the signal transmitter. If the telecommunication coverage is weak or non-existent, the cost of the transfer trip scheme alone could kill a DG project [5]. But Transfer trip scheme can be an effective and simple method for islanding prevention for distribution feeders with fixed topology [6].

\subsection{Power line signaling scheme}

Power line signaling scheme is similar to the telecomm-based transfer trip scheme. However, the signal is sent through the power line, which makes the scheme applicable to any distribution systems regardless the availability of telecomm means. Figure .1 shows a power line signaling scheme. This scheme can be effectively used in muti DG systems.

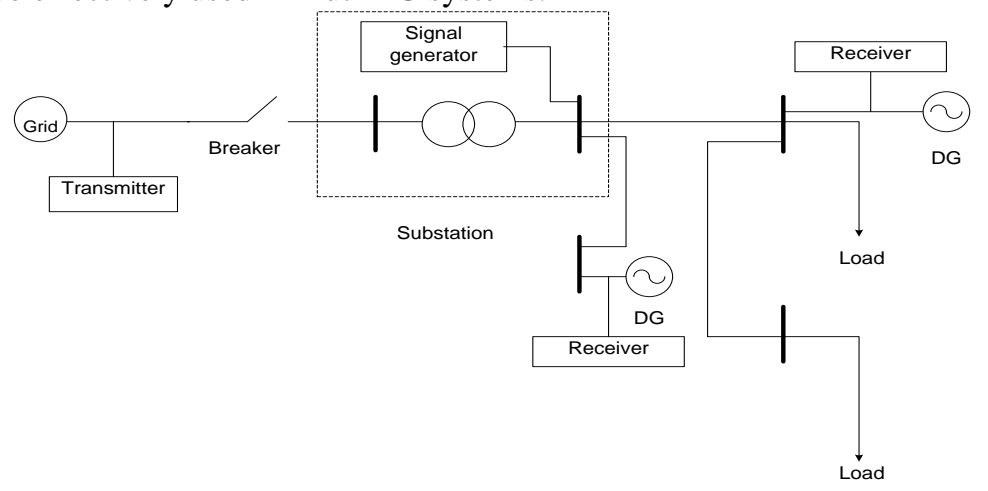

Fig.1. A power line signaling scheme

This scheme includes two devices: a signal generator which is connected to the substation bus and single receiver at the terminal of a given DG. The signal generator broadcasts a signal to all distribution feeders with a preset protocol continuously. If the detector of the DG does not sense the signal (caused by the opening of any devices between the substation and the DG) for certain duration, it is considered as an island condition and the DG can be tripped immediately. If 
the substation bus loses power, which is another islanding condition, the signal generator also loses power and stops broadcasting. So that downstream DGs will also trip. Furthermore, the signal generator has several auxiliary inputs. Any one of the inputs can stop the broadcast, resulting in tripping all DGs in the system. This feature is particularly useful when transmission system operators need to trip the DGs. It is also useful if a transmission system island is formed [7].

This scheme works like transfer trip scheme, but it works independent of network topology changes, the opening of any devices can be detected automatically since power line is used as signal carrier. It is economical comparing with transfer trip scheme due to the installation of one signal generator at the substation side which can satisfy the need of all downstream DGs. Besides without actually breaking up all the distribution feeders the scheme can be tested. However, this scheme has two main disadvantages. The first one is the cost of the signal generator. This is a medium voltage device. A step down transformer is required to connect it and it has to be installed in a substation. This cost may be hard to justify if there are only one or two DGs using the service. The second concern is the possible interference of the signal with other power line communication applications such as automatic meter reading. This is a promising technology but there is no field application experience of this technology yet [8].

\section{Local Detection Techniques}

Local detection techniques are based on the measurement of system parameters at the DG site. It is further divided into passive methods and active methods.

\subsection{Passive methods}

Passive methods are based on the information available on the DG site at the point of common coupling (PCC) with the utility grid. Passive anti-islanding techniques rely on the detection of abnormalities at the DG output when islanding occurs. Differentiation between an islanding and grid-connected condition is based upon to set the thresholds to detect islanding and prevent nuisance trips at the same time. However, it is usually difficult with passive techniques to set thresholds to prevent both islanding and nuisance trips at the same time. Conversely, the effectiveness of passive methods is not guaranteed for all loading conditions, in particular when there is a balanced DGs source [910]. Comparing with active method, there is a larger non-detection zone. But passive methods do not disturb the system.

Figure.2. shows the power circuit structure of DG connected to the utility grid with passive RLC loads. During the normal operation, because the values 
are controlled by the stiff grid with constant voltage and frequency, when the island occurs there is a mismatch between the DG power and local load power. The over/under voltage and frequency methods will not work properly to detect the island when the local loads closely match the DG output power. In this case the voltage and frequency shift are insufficient to exceed the voltage and frequency limits to allow the over/under voltage or frequency protection to trip. Since the distributed generation monitors the terminal voltage for its own control purpose; adding passive islanding detection usually requires little additional hardware and can be implemented at low cost [11].

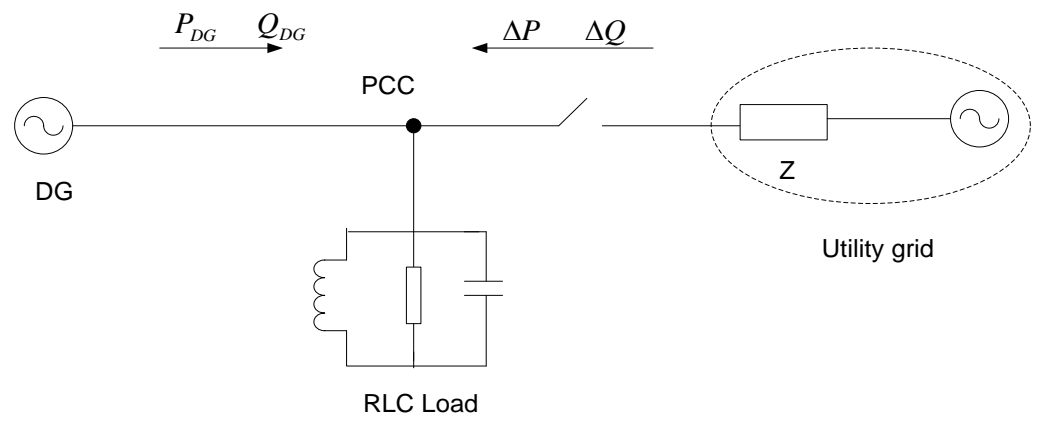

Fig.2. Power circuit structural of DG coupled to utility grid with RLC load

\subsection{Active methods}

With active methods, islanding can be detected even under conditions of perfect match between generation and load, which is not possible in case of the passive detection schemes. In active methods perturbations are directly introduced into the power system operation [12]. The principle of active method is that small perturbations will result in a significant change in system parameters when the DG is islanded. Active methods involve some kind of feedback technique or control mechanism that detects changes in the frequency or voltage at the PCC .

\subsection{Hybird}

Passive and active methods are two major categories of anti-islanding approaches which are being widely used in grid-connected distributed generation (DG) systems. Passive anti-islanding techniques have no negative impact on the inverter's performance however a large non-detection zone is the shortcoming of these techniques. On the other hand, active approaches have a smaller non-detection zone, but these inevitably have negative impact on system power quality. Hybrid methods based on combining the active and passive 
method are applied to solve these problems. The active method is implemented only when the islanding is suspected by the passive technique.

\section{Conclusion}

This paper provides many islanding detection methods, which can be broadly classified into remote and local techniques, local techniques are further divided into passive active and hybrid techniques. Each technique has its advantage and limitation. Comparison of these methods can provide useful information for determining a method to use, there is no single islanding detection technique which works satisfactorily for all systems under all situations. It is necessary to know the limitations these islanding methods possess before the comparison. The main considerations are:

- High implementation costs

- Susceptibility to false operation in multiple DG case

- Presence of NDZ's

- Reduction in power quality and system stability due to positive feedback

Choice of the islanding detection technique will largely depend on the type of the DG and system characteristics. Recently hybrid detection techniques have been proposed, compared with passive method, there is a small nondetection zone but longer detection time; the perturbation is introduced in the hybrid method only when an islanding situation is suspected unlike the active method. In paper [12-13] the combination of ROCOF relays with rate of change of power and interlock functions are introduced to detect islanding and avoid false tripping during non-islanding situation (such as load changing), compared with hybrid algorithm the detection time combination of two passive methods is much smaller and there is no perturbation injected into the system, which are the advantages over active and hybrid methods.

\section{References}

1. Chiradeja, P. Benefit of Distributed Generation: A Line Loss Reduction Analysis. in Transmission and Distribution Conference and Exhibition: Asia and Pacific, 2005 IEEE/PES. 2005.

2. Shahidehpour, M. and F. Schwarts, Don't let the sun go down on PV [photovoltaic systems]. Power and Energy Magazine, IEEE, 2004. 2(3): p. 40-48.

3. World Wind Energy Report 2010, G.E.C.D.i. Powerhouses, Editor. Nov,2011, 10th world wind energy conference and renewable energy exhibition. 
4. Wind Power Increase in 2008 Exceeds 10-year Average Growth Rate. Nov,2011; Available from: http://www.worldwatch.org/n ode $/ 6102$ ?emc $=e 1 \& m=239273 \& \mathrm{l}=5 \& \mathrm{v}=\mathrm{ca} 5 \mathrm{~d} 0 \mathrm{bd} 2 \mathrm{df}$.

5. BTM Forecasts 340-GW of Wind Energy by 2013. Dec,2011; Available from:http://www.renewableenergyworld.com/rea/news/article/2009/03/btmforecasts-340-gw-of-wind-by-2013? src=rss.

6. Global Status report. Dec,2011; Available from: http://www.ren21.net/Portals/97/documents/GSR/GSR2011_Master18.pdf.

7. Global Wind Report 2010. Dec,2011; Available from: http://www.gwec.net/index.php?id=180.

8. Solar Energy News. Dec,2011; Available from: http://www.brighterenergy.org/9715/news/solar/sunpower-offers-solarmodules-boasting-19-efficiency/.

9. Leading the solar energy revolution with Concentrator Photovoltaic (CPV) technology. Dec,2011; Available from: http://www.soitec.com/en/solarenergy/.

10. Strath, N., Islanding Detection in Power System. 2005, Lund University: SWEDEN.

11. Wilsun Xu, K.M., Sylvain Martel, An Assessment of Distributed Generation Islanding Detection Methods and Issues for Canada, C.E.T.C. (CETC), Editor. July 2004, Natrul Resource Canada.

12. Kunte, R.S. and W.Z. Gao, Comparison and Review of Islanding Detection Techniques for Distributed Energy Resources. 2008 40th North American Power Symposium (Naps 2008), 2008: p. 209-216.

13. Mahat, P., C. Zhe, and B. Bak-Jensen. Review of islanding detection methods for distributed generation. in Electric Utility Deregulation and Restructuring and Power Technologies, 2008. DRPT 2008. Third International Conference on. 2008. 